\title{
A stochastic version of the Noether Theorem
}

\author{
Alfredo González Lezcano ${ }^{a}$ and Alejandro Cabo Montes de Oca ${ }^{b}$ \\ a Departamento de Física, Universidad de Pinar del Río, \\ Pinar del Río, Cuba, \\ b Instituto de Matemática, Cibernética y Física, and \\ Calle E, No. 309, e/ 13 y 15, Vedado, La Habana, Cuba.
}

\begin{abstract}
A stochastic version of the Noether Theorem is derived for systems under the action of external random forces. The concept of moment generating functional is employed to describe the symmetry of the stochastic forces. The theorem is applied to two kinds of random covariant forces. One of them generated in an electrodynamic way and the other defined in the rest frame of the particle as a function of the proper time. For both of them, it is shown the conservation of the mean value of a random drift momentum. The validity of the theorem makes clear that random systems can produce causal stochastic correlations between two faraway separated systems, that had interacted in the past. In addition possible connections of the discussion with the Ives Couder's experimental results are remarked.
\end{abstract}

PACS numbers: 02.50.Ey; 03.65.Ud; 05.30.Ch; 05.40.-a

\section{INTRODUCTION}

The Noether theorem is an important result in theoretical physics and can be roughly stated as: If the Lagrangian of a physical system has a certain symmetry, then there is a conservation law for a quantity $\mathcal{J}$ called as the Noether's current [1-4]. Depending of the symmetry of the Lagrangian, the Noether current becomes angular momentum, linear momentum and energy, for spatial isotropy, spatial homogeneity and time invariance, respectively. Consider the dynamic system described by a Lagrangian $\mathcal{L}$ with certain symmetries. Then, according to the theorem, the corresponding currents will be conserved. In the presence of a generic external forces, this conservation law is not valid in general. If these external forces has some random behavior, then the question arise of whether it is possible to keep standing a conservation law at least for means values of the Noether currents. There are some situations where the influence of the stochastic field on the system is in certain sense symmetric. That is, for instance, in case that the spatial homogeneity, if somehow the field has the same influence in different points of the space. Stochastic forces are functions of the form $Q(q, \dot{q}, \theta)$ 13, 14], being $q, \dot{q}$ and $\theta$ a compact notation for a set of generalized coordinates, velocities and random variables, respectively. In reference [12] it was suggested the possibility of an extension of the Noether Theorem to a system of particles subject to the action of an external stochastic field. Simulations of one particle trajectories (and binary collisions for interacting particles) predicted the conservation of mean values for linear momentum. This conclusion was a considered to be a consequence of the relativistic invariance of the external force, which was defined in that work as a function of the proper time. The idea of the conservation of mean values of linear momentum for particles submitted to external forces was also suggested by a series of experiments recently carried out by Couder etal [8 [10]. In these works a situation consisting of a liquid droplet bouncing over a vibrating liquid surface was experimentally investigated. The measurements showed that the interaction of the droplet with the surface wave it creates, causes (under some specific experimental conditions) a drift movement with conserved mean velocity. The experiments also exhibited interesting properties strongly resembling the quantum behavior. The possibility of an extension of the Noether Theorem for systems sharing the main characteristic of being affected by stochastic forces is closely related with the symmetry properties of those forces. To speak about symmetry of a stochastic field could bring about some confusion, taking into account that the used transformations do not affect the random variables. That is: to impose symmetry conditions to the these forces can't be done directly on the function $Q(q, \dot{q}, \theta)$ because the stochastic character of the field avoids the possibility to predict the value of the variation for different settings of $\theta$. In order to be able to make a general extension of the Noether Theorem for this kind of systems, it will be employed a functional that, in analogy with the action of dynamic systems [1-4], can fully describe the stochastic process defining the forces and then impose symmetry conditions to this functional. It should remarked that a slightly different formulation of a stochastic Noether theorem was advanced in reference [11]. This work, basically discusses the cases of special Markov's random processes which seems to be much restricted than the here considered ones. The only constraint of the discussion here is that the physical system has equations of motions coming from a Lagrangian. Thus, we consider our discussion as being complementary to the one done in [11].

The analysis in general supports the possibility that two random classical systems which interact during a finite time lapse, and afterwards flight far apart, can retain correlations between their physical properties, which can describe 
apparent non causal links between physical quantities measured in the two well separated classical systems, after they stop to interact. This property seems to leave space for the explanation of EPR effects ([16]) in hidden variable theories, in a way satisfying the Bell restrictions about the existence of such models [15].

It also suggests the interest of coupling the "self-field" to stochastically driven particles as the ones discussed here. That is, to consider the existence at the particle place a point sources of the Klein-Gordon field, by example. In these cases, let us assume that a momentum conservation can be derived. Then, if a system of reference exists in which the momentum vanishes and the coupled particle-wave-modes show a spacially localized structure, it will imply that in this frame, the random distribution might describe a spatially localized stationary movements of the particle coupled with the stochastically populated self-field modes. But, assuming that the system is relativistic invariant, in a different Lorentz frame, they will exist analogous, but uniformly moving localized structures. Therefore, the situation is quite resembling the one in the mentioned Couder's experiments [8-10]. If these expected particle wave composite structures could show interference effects when approaching two slits in a wall, a connection of the Couder's experimental results with microscopic hidden variable structures could be found. We expect to consider these possibilities in extensions of this work.

In Section 2 the stochastic form of the Noether theorem is formulated and proved for a general class of stochastic forces. Next, Sections 3 and 4, considers its application to the above mentioned two physical systems, in proving that they allow for motions conserving a mean drift velocity of the particles. In the Summary the results are reviewed and few remarks on the possible extensions of the work are advanced.

\section{STOCHASTIC NOETHER THEOREM}

Let us consider a the dynamic system described by a Lagrangian $L$ depending of $N$ generalized coordinates $q_{i}$, $i=1, \ldots, N$ and their respective velocities $\dot{q}$, in which the point indicates the time derivative. For considering the presence of an external force acting on the system, we will use the recourse of including in the Lagrangian a term from which the force is derived. However, to find the Lagrangian terms determining a force is not always easy or possible. The search of those action terms is a difficult problem and it is not satisfactorily solved for a generic force. However, immediate and exacts solutions are known for the electromagnetic forces and many relevant physical systems. We will restrict our discussion to those cases.

Nevertheless, we will start the discussion without initially requiring that the forces are derived from a Lagrangian. Then, in general, the Euler-Lagrange equations of motion can be written in the form:

$$
\frac{\partial L}{\partial q_{i}}-\frac{d}{d \tau}\left(\frac{\partial L}{\partial \dot{q}_{i}}\right)=-Q_{i}\left(q_{i}, \dot{q}_{i}\right),
$$

where $Q_{i}\left(q_{i}, \dot{q}_{i}\right)$ are the generalized forces that act on the system and depend on both the coordinates and velocities. These explicit dependencies will be in general omitted for simplifying the notation. If the unperturbed by the force system has a certain symmetry, then the variation of the Lagrangian with respect to the change in the variables defined by the symmetry should vanish

$$
\delta L=\sum_{i}\left(\frac{\partial L}{\partial q_{i}} \delta q_{i}+\frac{\partial L}{\partial \dot{q}_{i}} \delta \dot{q}_{i}\right)=0 .
$$

In (2) $\delta q_{i}$ and $\delta \dot{q}_{i}$ are just the changes in the generalized coordinates, the variation of which makes the Lagrangian to remain invariant. Now, lets us consider the standard reasonings for the demonstration of the Noether theorem in usual situations [1], but in this case in connection with the inhomogeneous Euler-Lagrange equations (1). Thus, making use of the derivative of a product in (2) and also considering that the coordinate variations are done at fixed instant of time, that is:

$$
\delta \dot{q}_{i}=\frac{\partial}{\partial t} \delta q_{i}
$$

leads to:

$$
\sum_{i}\left(\left[\frac{\partial L}{\partial q_{i}}-\frac{d}{d \tau}\left(\frac{\partial L}{\partial \dot{q}_{i}}\right)\right] \delta q_{i}+\frac{d}{d \tau}\left(\frac{\partial L}{\partial \dot{q}_{i}} \delta q_{i}\right)\right)=0
$$

Then, from (11) results:

$$
\frac{d}{d \tau} \sum_{i}\left(\frac{\partial L}{\partial \dot{q}_{i}} \delta q_{i}\right)=\sum_{i} Q_{i} \delta q_{i}
$$


If the symmetry group $G$ associated to the unperturbed Lagrangian (the one omitting the random forces) has an infinitesimal transformation generated by $r_{\max }$ parameters $r=1,2, \ldots, r_{\max }$, the variations can be expressed as

$$
\delta q_{i}=\sum_{r} \varepsilon^{r} \Lambda_{i}^{r}(q)
$$

and their substitution in (4) leads to:

$$
\frac{d}{d \tau} \sum_{i}\left(\frac{\partial L}{\partial \dot{q}_{i}} \varepsilon^{r} \Lambda_{i}^{r}(q)\right)=\sum_{i, r} Q_{i} \varepsilon^{r} \Lambda_{i}^{r}(q)
$$

Assuming the independence of the parameters $\varepsilon^{r}$, it is obtained that:

$$
\begin{aligned}
\frac{d \mathcal{J}^{r}}{d \tau} & =\sum_{i} Q_{i} \Lambda_{i}^{r}(q), \quad r=1, \ldots, r_{\max } \\
\mathcal{J}^{r} & =\sum_{i} \frac{\partial L}{\partial \dot{q}_{i}} \Lambda_{i}^{r}(q)
\end{aligned}
$$

where $\mathcal{J}^{r}=\sum_{i} \frac{\partial L}{\partial \dot{q}_{i}} \Lambda_{i}^{r}(q)$ for the various values of the index $r$, are the Noether currents which will be conserved if the generalized forces vanish. Further, we will assume that the stochastic forces are derivable from a Lagrangian $L^{\text {ram }}$ as

$$
\begin{aligned}
Q_{i}\left(q_{i}, \dot{q}\right) & =\frac{\delta S^{r a m}\left[q_{i}, \dot{q}_{i}\right]}{\delta q_{i}(t)}=-\left(\frac{\partial L^{r a m}\left(q_{i}, \dot{q}_{i}\right)}{\partial q_{i}(t)}-\frac{\partial}{\partial t}\left(\frac{\partial L^{r a m}\left(q_{i}, \dot{q}_{i}\right)}{\partial \dot{q}_{i}(t)}\right)\right) \\
S^{r a m}\left[q_{i}, \dot{q}_{i}\right] & =\int d t L^{r a m}\left(q_{i}, \dot{q}_{i}\right)
\end{aligned}
$$

\section{A. The random forces}

Since we will discuss the situation in which the forces are random, let us now define those forces. The aleatory behavior will be introduced by the dependence of the forces not only of the generalized coordinates $q=\left\{q_{1}, q_{2}, \ldots, q_{i}, \ldots, q_{N}\right\}$ and their velocities, but also on a set of random variables $\theta \in \Theta$, being $\Theta$ a generic sample space. That is $Q_{i}=Q_{i}(q, \dot{q}, \theta)$. Then, assuming well defined initial conditions for the set of generalized coordinates $q\left(t_{0}\right)=q_{0}$ at an initial fixed instant of time $t_{0}$, we can define an ensemble of a large number of coordinate trajectories $q(t, \theta)$ all satisfying the initial conditions but following different courses as the time increases in dependence of the random value of the parameter $\theta$. Therefore, we will define a mean value of an arbitrary function $R\left(q_{1}(t, \theta),, \ldots, q_{N}(t, \theta) \mid\right.$ $\left.\dot{q}_{1}(t, \theta), \ldots, \dot{q}_{N}(t, \theta)\right)$ of a set of coordinates and their velocities taken at a fixed instant $t$, in the form

$$
\left.\left\langle R\left(q_{1}(t, \theta), \ldots, q_{N}(t, \theta) \mid \dot{q}_{1}(t, \theta), \ldots, \dot{q}_{N}(t, \theta)\right)\right\rangle_{\theta}=\int d \theta \rho(\theta) R\left(q_{1}(t, \theta), \ldots, q_{N}(t, \theta) \mid \dot{q}_{1}(t, \theta), \ldots, \dot{q}_{N}(t, \theta)\right)\right),
$$

where the integration runs over the space of parameters $\theta$ and $\rho(\theta)$ is the probability density in this space. It is also possible to define, for an arbitrary time $t$, the density of the ending values of the coordinates and velocities in the phase space of coordinates and velocities. For this purpose, consider the large number of $\theta$ values $N_{\theta}$ and the number of ending points $N_{b o x}(t)$ of the coordinates and velocities $\left(q_{i}, \dot{q}_{i}\right)$ which at the time $t$, are inside a box of the phase space of size $\prod_{i} \Delta q_{i} \Delta \dot{q}_{i}$. Then, the density of points in phase space can be defined as

$$
\rho\left(q_{1}(t, \theta), . ., q_{N}(t, \theta) ; \dot{q}_{1}(t, \theta), \ldots, \dot{q}_{N}(t, \theta)\right)=\frac{N_{b o x}(t)}{N_{\theta}} .
$$

This definition allows to express the mean value (8) in an alternative form as an integral of the quantity $R$ multiplied by the density in phase space $\rho$ as

$$
\begin{aligned}
\langle R\rangle_{\theta} & =\int d \theta \rho(\theta) R\left(q_{1}(t, \theta), . ., q_{N}(t, \theta) ; \dot{q}_{1}(t, \theta), \ldots, \dot{q}_{N}(t, \theta)\right) \\
& =\prod_{i}\left(\int\left(d q_{i} d \dot{q}_{i}\right) \rho\left(q_{1}(t, \theta), \ldots, q_{N}(t, \theta) ; \dot{q}_{1}(t, \theta), \ldots, \dot{q}_{N}(t, \theta)\right) R\left(q_{1}(t, \theta), \ldots, q_{N}(t, \theta) ; \dot{q}_{1}(t, \theta), \ldots, \dot{q}_{N}(t, \theta)\right) .\right.
\end{aligned}
$$


Now, if we assume that the generalized forces $Q_{i}$ are of the random kind defined above $Q_{i}=Q_{i}(q, \dot{q}, \theta)$ and take the mean value in both sides of the equation (6), it directly follows the relation

$$
\frac{d\left\langle\mathcal{J}^{r}\right\rangle_{\theta}}{d t}=\left\langle\sum_{i} Q_{i}(q(t, \theta), \dot{q}(t, \theta), \theta) \Lambda_{i}^{r}(q(t, \theta))\right\rangle_{\theta} .
$$

Therefore, the mean value of the quantity $\sum_{i} Q_{i}(q) \Lambda_{i}^{r}(q)$ is equal to the time variation of the Noether current of the unperturbed Lagrangian. In what follows we will consider the proof of the Noether theorem and the finding of a formula for their conserved currents by searching for a connection of the condition (12) with the symmetry properties of the random force distribution.

For the sake of definiteness let us precisely formulate the random Noether Theorem as follows:

If the stochastic forces derivable from a Lagrangian term share a certain symmetry with the Lagrangian of the system on which they act, then a conservation law of the stochastic mean value of a Noether current as a function of time is valid.

First of all, we must define what will be understood by a symmetry of the random forces. For this purpose we will make use of the property that a stochastic process is completely characterized by its moment (or characteristic) generating functional of the auxiliary variables $k=\left\{k_{1}(t), k_{2}(t), \ldots, k_{i}(t), \ldots, k_{N}(t)\right\}[13$, 14]:

$$
\Gamma[k]=\left\langle\exp \left(i \int_{-\infty}^{\infty} k(t) L^{r a m}(q(t, \theta), \dot{q}(t, \theta), \theta) d t\right)\right\rangle_{\theta},
$$

where $L^{r a m}$ is the previously defined action term generating the random forces. Then, we will consider that the symmetry of this functional over the changes of coordinate variables defined by the symmetry group is equivalent to the invariance of the corresponding stochastic force [13, 14]. Thus, if the symmetry group of $G$ is parametric with infinitesimal transformation $\delta q_{i}=\sum_{r} \varepsilon^{r} \Lambda_{i}^{r}(q), r=1, \ldots, r_{\max }$, then the increment of $\Gamma$ should exactly vanish when the symmetry coordinate change is done in (13) for arbitrary values of the auxiliary parameters $k$ and the group infinitesimal constants $\varepsilon^{r}$. Therefore

$$
\begin{aligned}
& \delta \Gamma[k]=\left\langle i \int_{-\infty}^{\infty} k\left(t^{\prime}\right) \delta L^{r a m}\left(q\left(t^{\prime}, \theta\right), \dot{q}\left(t^{\prime}, \theta\right), \theta\right) d t^{\prime} \times\right. \\
& \left.\exp \left(i \int_{-\infty}^{\infty} k\left(t^{\prime}\right) L^{r a m}\left(q\left(t^{\prime}, \theta\right), \dot{q}\left(t^{\prime}, \theta\right), \theta\right) d t^{\prime}\right)\right\rangle_{\theta} \\
& =\left\langle i \int_{-\infty}^{\infty} k\left(t^{\prime}\right) \sum_{i}\left(\frac{\partial L^{r a m}}{\partial q_{i}\left(t^{\prime}, \theta\right)} \delta q_{i}+\frac{\partial L^{r a m}}{\partial \dot{q}_{i}\left(t^{\prime}, \theta\right)} \frac{d}{d t^{\prime}} \delta q_{i}\right) d t^{\prime} \times\right. \\
& \left.\exp \left(i \int_{-\infty}^{\infty} k\left(t^{\prime}\right) L^{r a m}\left(q\left(t^{\prime}, \theta\right), \dot{q}\left(t^{\prime}, \theta\right), \theta\right) d t^{\prime}\right)\right\rangle_{\theta} \\
& =\left\langlei \int _ { - \infty } ^ { \infty } \sum _ { i , r } \left( k\left(t^{\prime}\right)\left(\frac{\partial L^{r a m}}{\partial q_{i}\left(t^{\prime}, \theta\right)}-\frac{d}{d t}\left(\frac{\partial L^{r a m}}{\partial \dot{q}_{i}\left(t^{\prime}, \theta\right)}\right)\right) \varepsilon^{r} \Lambda_{i}^{r}\left(q\left(t^{\prime}, \theta\right)\right)+\right.\right. \\
& \frac{d}{d t^{\prime}}\left(k\left(t^{\prime}\right) \sum_{i, r} \frac{\partial L^{r a m}}{\partial \dot{q}_{i}\left(t^{\prime}, \theta\right)} \varepsilon^{r} \Lambda_{i}^{r}\left(q\left(t^{\prime}, \theta\right)\right)\right) d t^{\prime} \times \\
& \left.\exp \left(i \int_{-\infty}^{\infty} k\left(t^{\prime}\right) L^{r a m}\left(q\left(t^{\prime}, \theta\right), \dot{q}\left(t^{\prime}, \theta\right), \theta\right) d t^{\prime}\right)\right\rangle \\
& =0 \text {. }
\end{aligned}
$$

Then, after assuming that the text functions $k(t)$ tend to vanish when $t$ tends to $\pm \infty$, the following relations follow 
as conditions for the stochastic Lagrangian to be symmetric

$$
\begin{aligned}
& \left\langle\sum_{i, r}\left(\frac{\partial L^{r a m}}{\partial q_{i}(t, \theta)}-\frac{d}{d t} \frac{\partial L^{r a m}}{\partial \dot{q}_{i}(t, \theta)}\right) \Lambda_{i}^{r}(q(t, \theta))\right\rangle_{\theta} \\
& =\left\langle\sum_{i, r} Q_{i}(q(t, \theta), \dot{q}(t, \theta), \theta) \Lambda_{i}^{r}(q(t, \theta))\right\rangle_{\theta}=0, \\
& r=1, \ldots, r_{\max } .
\end{aligned}
$$

But, substituting (15) in (12)

$$
\begin{aligned}
\frac{d\left\langle\mathcal{J}^{r}\right\rangle_{\theta}}{d t} & =\left\langle\sum_{i} Q_{i}(q(t, \theta), \dot{q}(t, \theta), \theta) \Lambda_{i}^{r}(q(t, \theta))\right\rangle_{\theta} \\
& =0 .
\end{aligned}
$$

This expression indicates that the Noether currents $\left\langle\mathcal{J}^{r}\right\rangle_{\theta}$ are conserved in time

$$
\begin{aligned}
\frac{d}{d t}\left\langle\mathcal{J}^{r}\right\rangle_{\theta} & =0, \\
\mathcal{J}^{r}(q(t, \theta), \dot{q}(t, \theta)) & =\sum_{i} \frac{\partial L(q(t, \theta), \dot{q}(t, \theta))}{\partial \dot{q}_{i}(t, \theta)} \Lambda_{i}^{r}(q(t, \theta)) .
\end{aligned}
$$

This result finishes the proof of the stochastic Noether theorem and the finding of the formulae for the conserved currents.

\section{RANDOM ELECTRODYNAMIC FORCES}

Let us consider in this section the application of the former discussion to stochastic electrodynamic forces. This kind of forces had been considered in the literature within the theory known as Stochastic Quantum Electrodynamics (SQED) in which the vacuum is assumed to create a force on a charged particle which is generated by a specially defined stochastic "vacuum" electromagnetic field [5, 6]. To start, below we will give the definition of the relativistic stochastic process under consideration. The random character of the forces will be implemented as in the past section, by defining a large ensemble of particle trajectories space $\{x(t, \theta)\}$ were $x(t, \theta)$ is the position four vector of the particle as a function of time and the parameter $\theta$. Changing $\theta$ pertaining to the set $\Theta$ will produce the ensemble random trajectories $C(\theta)$. As it was mentioned, in this section the symbol $x$ will indicate a compact form for a four-vector $x^{\mu}=\left(x^{0}, \vec{x}\right)$, the time $t=x^{0}$ will be measured in $\mathrm{cm}$ and will be equal to the product of the light velocity in $\mathrm{cm} / \mathrm{s}$ and the time measured in seconds. Let us write now a Lagrangian from which the random equations of motions can derived in the form

$$
\begin{aligned}
L_{T} & =-m \sqrt{1-(\vec{v}(t, \theta))^{2}}-q\left(\phi(\vec{x}(t, \theta), t)-\vec{v}(t, \theta) \cdot \vec{A}(\vec{x}(t, \theta), t)=L+L_{r a m},\right. \\
L & =-m \sqrt{1-(\vec{v}(t, \theta))^{2}}, \quad L_{r a m}=-q(\phi(\vec{x}(t, \theta), t)-\vec{v}(t, \theta) \cdot \vec{A}(\vec{x}(t, \theta), t), \\
\vec{v}(t, \theta) & =\frac{d}{d t} \vec{x}(t, \theta), \quad x(t, \theta)=(t, \vec{x}(t, \theta)), \quad t=x_{0} .
\end{aligned}
$$

Each of the trajectories in the ensemble will be defined by a solution of the equation of motion given by the above Lagrangian [6]

$$
\begin{aligned}
\frac{d}{d t} p^{\mu}(t, \theta) & =\frac{q}{m} F_{\nu}^{\mu}(x(t, \theta)) \frac{d}{d t} x^{\nu}(t, \theta), \\
p^{\nu}(t, \theta) & =m u^{\mu}(t, \theta)=m \frac{d}{d \tau} x^{\nu}(t, \theta) \\
& =\frac{m}{\sqrt{1-(\vec{v}(t, \theta))^{2}}} \frac{d}{d t} x^{\nu}(t, \theta) \\
& =\frac{m}{\sqrt{1-(\vec{v}(t, \theta))^{2}}}\left(\begin{array}{c}
1 \\
\vec{v}(t, \theta)
\end{array}\right) .
\end{aligned}
$$


It should be noted that contracting these equations with $p^{\mu}(t)$ and using the anti-symmetry of the intensity tensor it follows

$$
p_{\mu}(t) \frac{d}{d t} p^{\mu}(t)=\frac{d}{d t} p^{2}(t)=0,
$$

which is a dynamic constraint. This implies that only three equations from the four ones are independent. That is, the temporal equation can be derived form the other three spatial ones

$$
\begin{aligned}
\frac{d}{d t} p^{i}(t, \theta) & =\frac{q}{m}\left(F_{j}^{i}(x(t, \theta), t) \frac{d}{d t} x^{j}(t, \theta)+F_{0}^{i}(x(t, \theta), t),\right. \\
i & =1,2,3 .
\end{aligned}
$$

The vector potential $A_{\beta}(x)$ and its field intensity $F_{\gamma \beta}$ are given by

$$
\begin{aligned}
F_{\gamma \beta}(x) & =\partial_{\gamma} A_{\beta}(x)-\partial_{\beta} A_{\gamma}(x), \\
A_{\beta}(x) & =(0, \vec{A}(x)), \\
\vec{A}(x) & =\sum_{\lambda=1}^{2} \int d \vec{k} \frac{1}{w_{k}} \vec{\epsilon}(\vec{k}, \lambda) h(\vec{k}, \lambda) \times \\
& \sin \left(\vec{k} \cdot \vec{x}-w_{k} x^{0}+\theta(\vec{k}, \lambda)\right), \\
w_{k} & =|\vec{k}|,
\end{aligned}
$$

where the electromagnetic field was chosen in the Coulomb gauge

$$
\vec{\nabla} \cdot \vec{A}(x)=0, \quad \phi(x)=0,
$$

and the $\vec{\epsilon}(\vec{k}, \lambda)$ are two unit polarization vectors associated to the wave vector $\vec{k}$ and satisfying

$$
\vec{\epsilon}(\vec{k}, \lambda) \cdot \vec{\epsilon}\left(\vec{k}, \lambda^{\prime}\right)=\delta_{\lambda \lambda^{\prime}}, \quad \vec{k} \cdot \vec{\epsilon}(\vec{k}, \lambda)=0 .
$$

The number $h$ is chosen as the value determining that each wave mode has a exactly one half of photon quantum energy $w_{k}: \pi^{2} h^{2}=\frac{1}{2} w_{\vec{k}}$. The metric tensor in this section is assumed as diagonal and having $-g_{00}=1=g_{11}=g_{22}=g_{33}$.

Further, the parameters $\theta \in \Theta$ are defined as the set of random phase functions $\theta=\{\theta(\vec{k}, \lambda)\}$ (one for each pair of momentum and polarization $(\vec{k}, \lambda))$ which are uniformly distributed in the interval $(-\pi, \pi)[\underline{6}]$. Then, the distribution function in the space of parameters and the volume deferential in this space will be defined by the following products

$$
\begin{aligned}
& \rho(\theta)=\prod_{(\vec{k}, \lambda)} \frac{\Theta(\pi-\theta(\vec{k}, \lambda)) \Theta(\theta(\vec{k}, \lambda)-\pi)}{2 \pi}, \\
& d \theta=\prod_{(\vec{k}, \lambda)} d \theta(\vec{k}, \lambda) .
\end{aligned}
$$

For the mean value of any function of the coordinates and velocities taken at a given time instant we write

$$
\left\langleR \left(\vec{x}(t, \theta)|\vec{v}(t, \theta)\rangle_{\theta}=\int d \theta \rho(\theta) R(\vec{x}(t, \theta) \mid \vec{v}(t, \theta)) .\right.\right.
$$

\section{A. Invariance under space translation}

Let us no consider the invariance transformation

$$
x^{i} \rightarrow x^{i}+\delta x^{i}, \quad \delta x^{i}=\epsilon^{i},
$$

where the $\epsilon^{i}$ are constant infinitesimal increments in the coordinates. It is clear that the instantaneous Lagrangian is not invariant under this transformations. However, the stochastic system, if its random properties do not depend on the spatial point in which are measured, should show a special form of this invariance. Let us argue that is the case, 
as indicated by the Noether theorem presented in the previous section. This form of invariance can expressed in the notation of the definitions of the previous sections with $i=r$ for $r=1,2,3$, that is $q_{i}=x^{i}, i=1,2,3$. Thus, for the function $\Lambda$ generating the transformation it follows

$$
\Lambda_{i}^{r}(q)=\delta_{i}^{r} \quad, \quad i=1,2,3 .
$$

The unperturbed $L$ and the random $L_{\text {ram }}$ Lagrangians can be written as

$$
\begin{aligned}
L(\vec{x}(t, \theta), \vec{v}(t, \theta)) & =-m \sqrt{1-(\vec{v}(t, \theta))^{2}}, \\
L_{r a m} & =q \frac{d}{d t} \vec{x}(t, \theta) \cdot \vec{A}(x(t, \theta) .
\end{aligned}
$$

Thus, the possibly conserved currents associated to the Noether theorem take the forms

$$
\begin{aligned}
\mathcal{J}^{r}(\vec{x}(t, \theta), \vec{v}(t, \theta)) & =\frac{\partial L}{\partial \frac{d}{d t} x^{i}(t, \theta)} \\
& =\frac{m v^{i}(t, \theta)}{\sqrt{1-(\vec{v}(t, \theta))^{2}}},
\end{aligned}
$$

Therefore, the satisfaction of the property derived in the previous section for the stochastic forces to be translation invariant will effectively define wether or not this charges will be conserved after taken their random mean values. These conditions take the specific forms

$$
\begin{aligned}
& \left\langle\sum_{i}\left(\frac{\partial L^{r a m}}{\partial x^{i}(t, \theta)}-\frac{d}{d t} \frac{\partial L^{r a m}}{\left(\frac{d}{d t} x^{i}(t, \theta)\right)}\right)\right\rangle_{\theta} \delta^{i r} \\
& =-\left\langle\sum_{i} Q_{i}(\vec{x}(t, \theta), \vec{v}(t, \theta))\right\rangle_{\theta} \delta^{i r}=0, \quad r=1,2,3,
\end{aligned}
$$

in which the generalized forces have the formulae

$$
\begin{aligned}
Q^{i}(\vec{x}(t, \theta), \vec{v}(t, \theta)) & =-\left(\frac{\partial L^{r a m}(\vec{x}(t, \theta), \vec{v}(t, \theta))}{\partial x_{i}(t, \theta)}-\frac{\partial}{\partial t}\left(\frac{\partial L^{r a m}(\vec{x}(t, \theta), \vec{v}(t, \theta))}{\partial \dot{x}_{i}(t, \theta)}\right)\right) \\
& =\frac{q}{m}\left(F_{j}^{i}(\vec{x}(t, \theta)) \vec{v}(t, \theta)+F_{0}^{i}(\vec{x}(t, \theta)),\right. \\
L_{r a m} & =q \vec{v}(t, \theta) \cdot \vec{A}(\vec{x}(t, \theta), t) .
\end{aligned}
$$

These conditions can be explicitly written as

$$
\left\langle Q^{i}\left(x(t, \theta), v^{j}(t, \theta)\right)\right\rangle_{\theta}=\left\langle\frac{q}{m}\left(F_{j}^{i}(x(t, \theta)) v^{j}(t, \theta)+F_{0}^{i}(x(t, \theta))\right\rangle_{\theta}=0, \quad i=1,2,3 .\right.
$$

in which, the field intensities are expressed as linear superpositions of space and time derivatives of the potential $A$. Thus, when the mode expansion of the potential is substituted, the result is a superposition of harmonic functions one for each mode. But, the time and spatial derivatives of those harmonic functions do not introduce phase functions outside their arguments. In this situation, let us assume that all but one of the phase functions are fixed and the integral over the single varying one is considered in (41). Then, since the number of modes is large, tending to infinity, the variation of a single phase, let say $\theta\left(\vec{k}_{o}, \lambda_{o}\right)$ should determine an infinitesimal variation of the trajectories and velocities $\vec{x}(t, \theta), \vec{v}(t, \theta)$. Thus, the integral over the phase $\theta\left(\vec{k}_{o}, \lambda\right)$ in the whole interval $(-\pi, \pi)$ will vanish because the integral in a period of any harmonic function is zero. The above argue can be alternatively illustrated by representing the measure of the random mean value as the product of the large number of phases which are kept constant and the integral over the single phase $\theta\left(\vec{k}_{o}, \lambda_{o}\right)$

$$
\begin{gathered}
\left(\prod_{\left(\overrightarrow{k^{\prime}}, \lambda^{\prime}\right)} d \theta\left(\overrightarrow{k^{\prime}}, \lambda^{\prime}\right) \Theta\left(\pi-\theta\left(\overrightarrow{k^{\prime}}, \lambda^{\prime}\right)\right) \Theta\left(\theta\left(\overrightarrow{k^{\prime}}, \lambda^{\prime}\right)-\pi\right)\right) \times \\
\int_{-\pi}^{\pi} d \theta\left(\vec{k}_{o}, \lambda_{o}\right) \sin \left(\vec{k} \cdot \vec{x}(t, \theta)-w_{k} x^{0}+\theta\left(\overrightarrow{k_{o}}, \lambda_{o}\right)\right) .
\end{gathered}
$$


Since all but one phase are fixed, the changes in the random trajectories $\vec{x}(t, \theta)$ defined by the variation of $\theta\left(\vec{k}_{o}, \lambda_{o}\right)$ in the integration should be vanishingly small and then $\vec{x}(t, \theta)$ can be considered as a constant in the integration. Thus the contribution of each mode to the expression of $Q^{i}$ in (41) vanishes.

In conclusion, the conditions for the validity of the translation invariance of the stochastic forces are obeyed

$$
\left\langle Q^{i}(\vec{x}(t, \theta), \vec{v}(t, \theta))\right\rangle_{\theta}=0
$$

and these relations imply the following drift momentum conservation laws

$$
\frac{d}{d t}\left\langle\mathcal{J}^{i}(\vec{x}(t, \theta), \vec{v}(t, \theta))\right\rangle_{\theta}=\frac{d}{d t}\left\langle\frac{m v^{i}(t, \theta)}{\sqrt{1-(\vec{v}(t, \theta))^{2}}}\right\rangle_{\theta}=0 .
$$

They indicate that the movement of a particle driven by these stochastic forces can show a constant mean momentum, as it should be in the case that the SQED theory is Lorentz invariant [5, 6].

\section{COVARIANT RANDOM FORCES}

In reference [12] it was studied the action a covariantly defined random force, by giving its expression in the rest system of a particle as a function of the proper time. Numerically, it was shown that the system can exhibit conserved drift velocities in spite of its random oscillation. The problem was discussed in one time and one space dimension, but the analysis might be extended to four dimensions. In this section, let us discuss this problem in the 2D Minkowski metric in which the components of the vector potential, the position vector and the metric are defined as

$$
\begin{aligned}
x_{\mu} & =\left(x_{1}, x_{2}\right)=\left(x_{1}, i x_{0}\right), . . g_{\mu \nu}=\delta_{\mu \nu} \\
c & =1, \quad t=x_{0},
\end{aligned}
$$

where the imaginary variable $x_{2}$, is analogous to the $x_{4}$ in four dimensional space. Let us consider the equation of motion of the particle, which in the laboratory frame is driven by a vacuum force defined by its value in the rest system of the particle, as a function of the proper time. This equation of motion can be written in the form

$$
\begin{aligned}
\frac{d p^{\mu}(\tau)}{d \tau} & =\frac{1}{i} \epsilon^{\mu \nu} \frac{p^{\nu}(\tau)}{m} \mathcal{F}(\tau), \\
\epsilon^{\mu \nu} & =\left\{\begin{array}{cc}
0, & \mu=\nu, \\
1, & \mu=1, \nu=2, \\
-1, & \mu=2, \nu=1,
\end{array}\right.
\end{aligned}
$$

in which $\epsilon^{\mu \nu}$ is the fully anti-symmetric tensor in two dimensions, and the appearing 2D-momentum, 2D-velocity and the proper time of the particle as a function of the usual time, are defined as

$$
\begin{aligned}
p^{\mu}(\tau) & =m u^{\mu}(\tau) \\
u^{\mu}(\tau) & =\frac{d x^{\mu}(\tau)}{d \tau} \\
\tau(t) & =\int_{-\infty}^{\infty} d t \sqrt{-\frac{d x^{\mu}(\tau)}{d t} \frac{d x^{\mu}(\tau)}{d t}} \\
& =\int_{-\infty}^{\infty} d t \sqrt{1-v_{1}^{2}(t)} \\
v_{1}(t) & =\frac{d x_{1}(t)}{d t} .
\end{aligned}
$$

The stochastic nature of the problem is introduced by the random scalar function of the proper time $\mathcal{F}(\tau, \theta))$. It is defined as a harmonic function of the proper time depending of the stochastic parameter $\theta$ in a similar way as in the past section, in the form

$$
\mathcal{F}(\tau, \theta)=\sum_{n=-\infty}^{\infty} \frac{2 \pi}{T} \exp \left(-i\left(w_{n} \tau+\theta_{w_{n}}\right)\right) S\left(w_{n}\right)
$$


where $T$ is a large proper time interval to be taken arbitrary large but finite, and the mode frequencies $\left\{w_{n}\right\}$ are defined as

$$
w_{n}=\frac{2 \pi}{T} n, \quad n=-\infty, \ldots-1,0,1, \ldots \infty .
$$

Further, the function $S\left(w_{n}\right)$ specifies a spectral weight for the proper time frequencies in which the force is expanded. Finally, for each frequency $w_{n}$ the stochastic element in the force is introduced in a similar form as it is in SQED: that is, by adding aleatory phase functions $\theta_{w_{n}}$ in the argument of each harmonic function, which is uniformly distributed in the interval $(-\pi, \pi)$. Again, the stochastic parameter $\theta$ is defined as the set $\theta=\left\{\theta_{w_{n}}\right\}$ of all the stochastic phases (one for each mode frequency $w_{n}$ ) and the probability density and the volume differential in the space of parameters as

$$
\begin{aligned}
\rho(\theta) & =\prod_{\left(w_{n}\right)} \frac{\left.\Theta\left(\pi-\theta_{w_{n}}\right)\right) \Theta\left(\theta_{w_{n}}-\pi\right)}{2 \pi}, \\
d \theta & =\prod_{\left(w_{n}\right)} d \theta_{w_{n}} .
\end{aligned}
$$

For the mean value of any function of the coordinates and velocities taken at a fixed laboratory frame time $t$ we have

$$
\begin{aligned}
& \left\langle R\left(x_{1}(t, \theta) \mid \dot{x_{1}}(t, \theta)\right)\right\rangle_{\theta} \\
& =\int d \theta \rho(\theta) R\left(x_{1}(t, \theta) \mid \dot{x_{1}}(t, \theta)\right), \\
& =\prod_{\left(w_{n}\right)}\left(\int_{-\pi}^{\pi} d \theta\left(w_{n}\right) \frac{\left.\Theta\left(\pi-\theta_{w_{n}}\right)\right) \Theta\left(\theta_{w_{n}}-\pi\right)}{2 \pi}\right) \times \\
& R\left(x_{1}(t, \theta) \mid \dot{x_{1}}(t, \theta)\right) .
\end{aligned}
$$

\section{A. Lagrangian formulation}

Since the problem is relativistically invariant the Lagrangian formulation becomes constrained, as it can be seen by contracting equation (45) with the 2D-momentum, which gives

$$
\begin{aligned}
p^{\mu}(\tau) \frac{d p^{\mu}(\tau)}{d \tau} & =\frac{1}{i} \epsilon^{\mu \nu} \frac{p^{\mu}(\tau) p^{\nu}(\tau)}{m} \mathcal{F}(\tau) \\
& =0=\frac{d p^{2}(\tau)}{d \tau}, \\
p^{2}(\tau) & =c t c .
\end{aligned}
$$

In order to find an unconstrained Lagrangian for the description of the system, we can consider the single spatial component of the equations (45), which can be written as

$$
\begin{aligned}
\frac{d p^{1}(\tau)}{d \tau} & =\frac{1}{i} \epsilon^{12} \frac{p^{2}(\tau)}{m} \mathcal{F}(\tau), \\
\frac{d p^{1}(t)}{\sqrt{1-v^{2}} d t} & =\frac{1}{\sqrt{1-v^{2}}} \mathcal{F}(\tau), \\
\frac{d}{d t} \frac{m v_{1}(t)}{\sqrt{1-v_{1}^{2}}} & =\mathcal{F}(\tau), \\
v_{1}(t) & =\frac{d x_{1}(t)}{d t} .
\end{aligned}
$$


The solution of this equation implies the satisfaction of the covariant ones (45). Thus the full dynamics is defined by it. But, this single equation can be derived from the Lagrangian

$$
\begin{aligned}
L_{\text {total }}\left(x(t, \theta), \frac{d x(t, \theta)}{d t}\right) & =-m \sqrt{1-\left(\frac{d x(t, \theta)}{d t}\right)^{2}}-\frac{d x(t, \theta)}{d t} \int_{-\infty}^{t} d t^{\prime} \mathcal{F}\left(\tau\left(t^{\prime}\right)\right) \\
& =L\left(x(t, \theta), \frac{d x(t, \theta)}{d t}\right)+L_{\text {ram }}\left(x(t, \theta), \frac{d x(t, \theta)}{d t}\right),
\end{aligned}
$$

where $\tau(t)$ is the proper time as a function of the laboratory frame time $t$ given in (49). Note in the above expression and in what follows in this section, that we have redefined the spatial coordinate of the particle by omitting the subindex 1 as

$$
x(t, \theta) \equiv x_{1}(t, \theta),
$$

in order to simplify the notation. Then, after considering the randomly generated trajectories $\{x(t, \theta)\}$ solving the Lagrange equation of motion

$$
\frac{d}{d t} \frac{m \frac{d x(t, \theta)}{d t}}{\sqrt{1-\left(\frac{d x(t, \theta)}{d t}\right)^{2}}}=\mathcal{F}(\tau(t, \theta)),
$$

and considering that the Lagrangian is independent of the space translations $x \rightarrow x+\delta a$, the stochastically conserved Noether current (20), which can exist in the case that the stochastic forces also obey their proper invariance condition (15), has the form

$$
\begin{aligned}
\mathcal{J}^{r}(x(t, \theta), \dot{x}(t, \theta)) & =\frac{\partial L(x(t, \theta), \dot{x}(t, \theta))}{\partial \dot{x}(t, \theta)} \\
& =\frac{m \frac{d x(t, \theta)}{d t}}{\sqrt{1-\left(\frac{d x(t, \theta)}{d t}\right)^{2}}} .
\end{aligned}
$$

Therefore, let us inspect the satisfaction of the invariance condition for the stochastic forces, which in this case takes the form

$$
\begin{aligned}
& \left\langle\left(\frac{\partial L^{r a m}}{\partial x(t, \theta)}-\frac{d}{d t} \frac{\partial L^{r a m}}{\partial \dot{x}(t, \theta)}\right)\right\rangle_{\theta} \\
& =\langle\mathcal{F}(\tau(t, \theta))\rangle_{\theta}=0 .
\end{aligned}
$$

Now, from the definition of the stochastic force, its mean value can be written in the form

$$
\begin{aligned}
\langle\mathcal{F}(\tau(t, \theta))\rangle_{\theta}= & \prod_{\left(w_{n}\right)}\left(\int_{-\pi}^{\pi} d \theta\left(w_{n}\right) \frac{\left.\Theta\left(\pi-\theta_{w_{n}}\right)\right) \Theta\left(\theta_{w_{n}}-\pi\right)}{2 \pi}\right) \times \\
& \sum_{n^{\prime}=-\infty}^{\infty} \frac{2 \pi}{T} \exp \left(-i\left(w_{n^{\prime}} \tau(t, \theta)+\theta_{w_{n^{\prime}}}\right)\right) G\left(w_{n^{\prime}}\right) .
\end{aligned}
$$

Then, similarly as it was done in the previous section, it is then possible to consider a differential region in the stochastic parameter space, taken around a fixed well defined values of the large number of the phase parameters associated to each mode $\left(\theta_{w_{1}} \ldots \theta_{w_{n}} \ldots\right)$. and integrate it over the values of a single mode phase factor $\theta_{w_{n^{\prime}}}$ over its region of definition $(-\pi, \pi)$. The contribution of a single mode $w_{n^{\prime}}$ to this quantity leads to the expression

$$
\begin{aligned}
& =\prod_{\left(w_{n} \neq w_{n^{\prime}}\right)}\left(d \theta\left(w_{n}\right) \frac{\left.\Theta\left(\pi-\theta_{w_{n}}\right)\right) \Theta\left(\theta_{w_{n}}-\pi\right)}{2 \pi}\right) \times \\
& \frac{2 \pi}{T} \int_{-\pi}^{\pi} \theta_{w_{n^{\prime}}} \exp \left(-i\left(w_{n^{\prime}} \tau(t, \theta)+\theta_{w_{n^{\prime}}}\right)\right) G\left(w_{n^{\prime}}\right) .
\end{aligned}
$$

Note that the whole mean values is a summation over $n^{\prime}$ and the integration over the rest of the large number of phase factors of such terms. Now, it can be noted that varying the phase of a single mode (within the large number of 
them approximating a continuum) should change in a differential form the expression of the proper time as a function of the laboratory frame time $\tau(t, \theta)$. Therefore, this function can be assumed as being constant in the integration over $\theta_{w_{n^{\prime}}}$. This property in turns implies the vanishing the mean value

$$
\langle\mathcal{F}(\tau(t, \theta))\rangle_{\theta}=0
$$

Therefore, the satisfaction of the condition for the stochastic invariance of the random forces implies the conservation of the linear momentum

$$
\frac{d}{d t}\left\langle\frac{m \frac{d x(t, \theta)}{d t}}{\sqrt{1-\left(\frac{d x(t, \theta)}{d t}\right)^{2}}}\right\rangle_{\theta}=0
$$

a conservation law, which due to the Lorentz covariance of the discussion also implies the energy constancy with time

$$
\frac{d}{d t}\left\langle\frac{1}{\sqrt{1-\left(\frac{d x(t, \theta)}{d t}\right)^{2}}}\right\rangle_{\theta}=0 .
$$

Thus, the time invariance of a drift four-momentum is implied by the stochastic form the Noether theorem also in this case.

\section{Summary}

A generalized version of the Noether theorem is proven which extends this property for Lagrangian systems in which stochastic forces are present. It shows that there are conserved in time functions of the coordinates and velocities, when the stochastic forces have symmetries which coincide with the invariances of the unperturbed Lagrangian (the non stochastic part of the Lagrangian). The considered forces are of general kind including coordinate as well as velocity dependencies. A characterization of the meaning of the symmetry of the random forces and the definition of the stochastic mean values being appropriate to formulate the conservation laws, are also given. The symmetry of the random forces are defined by the invariance of the moment generating functionals of their random Lagrangian with respect to a symmetry change of variables. The expression for the conserved Noether charges are derived. The results are applied to the equation of motion of the particles in Stochastic Quantum Electrodynamics [6, 7] and to the covariant random forces discussed in [12]. In both cases it follows that the mean value over the ensemble of the relativistic spatial momentum of the particles conserves in time [7].

The analysis also indicates the possibility that two random classical systems which interact during a finite time lapse, and afterwards flight far a apart, can retain correlations between their physical quantities. This property may describe apparent non causal correlations, existing between physical quantities measured in the two well separated classical systems after they stop to interact. This property, opens possibilities for the description of EPR effects ([16]) in hidden variable theories, being able to satisfy the Bell's constraints about the existence of such theories [15].

The presentation also suggests the interest in coupling a "self-field" to stochastically driven particles as the ones discussed here. Assuming that a momentum conservation might be derived' for these systems, and also given that a reference frame exists in which the momentum vanishes, the random distribution could describe spatially localized stationary movements of the particle coupled with the self-field wave modes. Then, if the system is relativistic invariant, in uniformly moving Lorentz frames localized random structures will exists showing constant drift velocities. This situation is quite resembling the one in the mentioned Couder's experiments [8 10]. Therefore, these expected particle wave composite structures have the chance of showing interference effects when approaching to two slits in a wall. Such a possibility suggests a connection of the Couder's experimental mechanical results with microscopic hidden variable theories. We expect to consider these possibilities in extensions of this work.

\section{Acknowledgments}

The support granted by the N-35 OEA Network of the ICTP is greatly appreciated.

[1] N. N. Bogoliubov, D. V. Shirkov, Introduction to the theory of quantized fields, Editorial Nauka (1980). 
[2] H. Goldstein, C. Poole and J. Safko, Classical mechanics, Addison-Wesley, Third Edition (2000).

[3] A. A. Sokolov, I. M. Ternov, Electrodinamica euantica, Ed. Mir Moscu (1989).

[4] L. D. Landau, E. M. Lifshitz, Teoria clasica de campos, Vol 2, Ch. 10. (1992).

[5] T. W. Marshall, Proc. R. Soc. A276, 475 (1963).

[6] T. Boyer, Phys.Rev. D 11, 790 (1975).

[7] L. de La Peña, A. M. Cetto, The quantum dice, ISBN 978-94-015-8723-5 (eBook), Alwyn van der Merwe, (1996).

[8] Y. Couder, S. Protiere, E. Fort and A. Boudaoud, Nature 437,208 (2005).

[9] S. Protiere, A. Boudaoud and Y. Couder, Fluid Mech. 554, 85 (2006).

[10] A. Eddi, E. Fort, F. Moisy and Y. Couder, Phys. Rev. Lett. 102, 240401 (2009).

[11] J. Baez and B. Fong, J. Math. Phys. 54 , 013301 (2013). arXiv:1203.2035

[12] A. Cabo-Bizet, A. Cabo Montes de Oca, Phys. Lett. A. 359, 265 (2006).

[13] N. G. van Kampen, Stochastics processes in physics and chemistry, Elsevier, Third Edition (2007).

[14] M. O. Cáceres, Elementos de estadística del no equilibrio. y procesos estocásticos, Editorial Revertè (2003).

[15] J. S. Bell, Rev. Mod. Phys. 38, 447 (1966).

[16] A. Einstein, B. Podolsky and N. Rosen, Phys. Rev. 47, 777 (1935). 